\title{
Enhancing Auditory Information - Implications for Catching Behaviour in Skilled Performers
}

\author{
Andrea Utley, M. Nasr, and A. Astill
}

This study aimed to examine the effect of enhancing perceptual information by using broadband sound to facilitate one-handed catching. Enhancing environmental information affects the perception-action coupling cycle (Newell, 1991), thus improving catching success and allowing greater flexibility of movement. Employing broadband sound in a ball provides bimodal stimuli that are spatially co-incidental and therefore enhancing sensory information (Stein, Meredith, Huneycutt, and Mcdade 1989). Twenty-two adults (M age 24) volunteered for the study, using $3 D$ kinematic analyses all participants were examined performing 30 one handed catches across three testing blocks. Analyses showed that enhancing auditory information with broadband noise resulted in a greater affordance for action. Data showed that by augmenting perceptual information, less time was required for the deceleration phase of the catch. Through analyses of the intralimb correlation, an indication of the presence of coordinative structures, it was found that participants with enhanced auditory information were able to 'play' with their movement patterns in order to achieve the task goal.

Keywords: Coordinative structures, joint coupling, broadband noise, one-handed catching

$\mathbf{T}$ he act of catching a ball has attracted considerable attention. It allows researchers to study the complex interactions between human motor control processes, whole body movements and the dynamic environment in which we live (Tresilian, 1999). Catching requires the pick up of predictive information, advance timing skills and correct spatial positioning of the hand in response to the ball. Previous studies have investigated 'catching behaviour' in order to discover the amount, or nature of information necessary to successfully catch a ball with one hand (Button, 2002, Fischman, Moore, and Steele 1992). Literature to date has largely focused on the role of visual information as a constraint on co-ordination, there has been little investigation into the effect of enhancing auditory information on coordination. The present study attempts to investigate a prior

Andrea Utley, Director Centre for Sport and Exercise Sciences, Senior Lecturer in Motor Control and Learning, University of Leeds, United Kingdom, M. Nasr and A. Astill are also associated with the same Centre. suggestion that auditory information could act as a significant constraint on co-ordinating interceptive tasks (Savelsbergh, Netelenbos \& Whiting, 1991).

Through the analysis of catching behaviour, which is a spatially and temporally constrained action of a precise nature (Alderson, Sully \& Sully, 1974), information pertaining to the movement coordination of performers can be assessed; and in particular, the ability of the body to overcome constraints and move the hand to the correct place in order to intercept a ball. A 'dynamic systems' approach to motor control and coordination examines the interaction between demands made by internal constraints such as body mechanics and the surrounding environment (Bernstein 1967). Bernstein (1967) highlighted the need to overcome the degrees of freedom 'problem'. That is, the control of the overwhelming number of independent variables without the need for an executive subsystem. Bernstein proposed a three-stage model of learning through which the degrees of freedom 'problem' can be solved: 
1. Freezing the number of degrees of freedom to a minimum

2. Gradual releasing of the degrees of freedom to incorporate movement coordination between the degrees of freedom

3. Reorganising the degrees of freedom into an economical movement pattern

The freezing stage reduces the number of degrees of freedom at the periphery that the central control mechanism must organise. This initial stage of Bernstein's hypothesis is highly task dependent, influenced by task difficulty, and different extents of freezing will be seen (Vereijken, van Emmerick, Whiting, and Newell). The freezing is achieved by the muscle and muscle groups being constrained to act as a functional unit. These functional units have been termed coordinative structures and are able to automatically adjust to the changing environment in order to achieve the same task goal (Turvey, 1977). A study of one-handed catching in children has illustrated that in early learning, poor catchers freeze their degrees of freedom; this is seen in the tightly coupled shoulder and elbow joints (Davids, Bennett, Court, Tayler \& Button, 1997 and Button, Davids, Bennett, Tayler 2000).

According to Bernstein, improvement in skill is characterized by a gradual releasing of the degrees of freedom. This is seen in two stages, firstly the incorporation of all possible degrees of freedom into coordinative structures, this then enables the individual to structure movements so as to, 'utilize entirely the reactive phenomena' which arise (Bernstein, 1967). In the same catching study, individual profiling of a skilled catcher illustrates that the shoulder, elbow and wrist joints are operating relatively independently and that flexibility in the coordination of the shoulder and elbow joints emerge (Davids et al, 1997).

Advocates of the dynamical systems approach to motor control and learning state that an inseparable coupling of perception and action is vital during the acquisition of new skills. Inexperienced movers will often attempt many different movement patterns in order to succeed. Practice is a continuous search for movement solutions within the perceptuo-motor works space, the constraints of which are provided by the interaction of the learner with the environment (Bennett, Davids, and Woodcock 1999). Throughout this process the temporary state of coordination becomes more resistant to the environmental forces which could perturb the stability of the system.

Few studies have addressed how the manipulation of the availability of auditory information during practice affects the acquisition of a motor skill, research to date has predominantly focused upon the removal and degradation of visual information. A study conducted by Button (2002) perturbed auditory information in a catching task by eliminating the noise produced by the ball machine on ball ejection. The most consistent observations obtained from this experiment were that skilled catchers required an increased peak wrist velocity and opening velocity of the hand to achieve the task goal. This illustrates that skilled catchers are able to alter well established perception action couplings and are able to make use of different types of information to support catching behaviour as environmental conditions change. The kinematic analyses of co-ordination patterns in this study highlighted the flexibility of skilled performers; as performance conditions change they can reassemble the perception-action couplings in order to achieve the task goal. Utley and Astill (2007) and Utley et al (2007) found that children without developmental coordination disorder were better able re-assemble their upper limbs when catching. This indicated a higher level of expertise. Chen and Yen (2009) also considered and found benefits of multisensory enhancement during fast movements.

\section{Broadband Noise and the Superior Colliculus}

The superior colliculus is a midbrain structure which receives visual, auditory and somatosensory input and is involved in multisensory integration (Sterbing, Hartung \& Hoffmann, 2002). It has been proposed that if both auditory and visual stimuli originate from the same source in sensory space, then an 
'integrated multisensory-multimotor' map is formed. Conversely, when an auditory stimulus is presented outside of its receptive field, the effectiveness of the visual stimulus is degraded (Stein \& Meredith, 1993). Neurophysiological studies have illustrated that bimodal cells within the superior colliculus can integrate audio and visual stimuli by overlapping their receptive fields (Lewald, Ehrenstein \& Guski 2001).

Knudsen, du Lac \& Esterley (1987), recognised that localising a sound requires a great deal of neural processing. Only certain types of sounds are inherently localizable and what is crucial is that they contain a large spectrum of frequencies that is broadband noise. Pure tones, simple tone combinations or narrowband noise cannot be localized. To understand why this is the case, the cues given by sound, recognised by the brain, must be considered. We can hear a vast range of frequencies, from approximately $20 \mathrm{~Hz}$ to $20 \mathrm{kHz}$, although this range diminishes as we age. There are three main types of information that allow the brain to localize sound. The first two are known as binaural cues because they make use of the fact that we have two ears, separated by the width of our head.

The final piece of information processed by the brain regarding sound localization is called the head-related transfer function (HRTF) (Carlile and King, 1993). The HRTF refers to the effect the external ear has on sound. As a result of passing over the bumps or convolutions of the pinnae, the sound is modified so that some frequencies are attenuated and others are amplified. Although there are certain generalities in the way the sound is modified by the pinnae, the HRTF of any one person is unique to that individual. The role of the HRTF is particularly important when we are trying to determine whether a sound is immediately in front of, or directly behind, us. In this instance the timing and intensity differences are negligible and there is consequently very little information available to the central nervous system on which to base a decision of 'in front' or 'behind'. Thus, to accurately locate the direction of a sound source and to overcome the ambiguities inherent to single tones, a broader frequency is used. Therefore, the source of

broadband noise which consists of a broader frequency is easily located in space. Utley, Nasr, Astill (2010) found that catching skills and the retention of catching skills improved in children with and without movement difficulties when practicing catching skills with a ball emitting broadband sound.

In light of the above information and the assumptions made by the theory of perceptionaction coupling it seems that augmenting auditory information in a catching task would result in a more successful catching performance and possibly a more adaptable movement pattern when using skilled catchers as participants. The purpose of the present study was to examine whether broadband noise, presented spatially and temporally coincident, would improve motor output by enhancing the formation of coordinative structures. Catching ability was both qualitatively and quantitatively assessed across trials by measuring the velocity of the catching arm and the correlation between the velocities of the joints. It was hypothesized that by augmenting auditory information of the experimental group, they would have a greater affordance for action than the control group, who did not have additional auditory stimuli. This would lead to a greater improvement in catching performance and stronger intralimb coupling which is an indication of the formation of coordinative structures. A greater affordance for action would also allow participants to have more flexibility to experiment with their responses to achieve a task goal.

\section{Method}

\section{Participants}

Twenty-two students from the University of Leeds volunteered to participate in this experiment, 9 males and 11 females $(M=24$, $S D=3.2)$. All participants reported normal, or corrected to normal vision and reported no movement impairments which could confound results.

\section{Apparatus}


Data was collected using the Kinemetrix 3D motion analysis system. Every catching attempt was measured in each experimental block of trials at a sampling rate of $50 \mathrm{~Hz}$, using 3 CCTV cameras with infrared band pass filters (see figure 2). During data collection a threshold level was set to reduce noise; other than this no filter or spline was utilized. Prior to each period of recording the system was calibrated. The error in measurement of the Kinemetrix system was maintained below $3.0 \mathrm{~mm}$ in the $\mathrm{x}, \mathrm{y}$ and $\mathrm{z}$ directions. Each block of trials was also recorded using a video recorder.

In order to collect data on joint positioning, luminous lightweight spheres $(1 \mathrm{~cm}$ or $2 \mathrm{~cm}$ diameter) were placed on the anterior aspect of the wrist $(1 \mathrm{~cm})$, elbow $(2 \mathrm{~cm})$ and gleno-humeral joint $(2 \mathrm{~cm})$ of the non-dominant arm. These anatomical locations were chosen as they can be palpably located and have been used in previous studies of one handed catching (Button, 2002).

A Bola Ball (Stuart \& Williams, Bristol, UK) ball projection machine was set up $4 \mathrm{~m}$ from participants to deliver balls at fifteen second intervals to the subjects at a speed of approximately $8 \mathrm{~ms}^{-1}$ (see figure 1), Balls were aimed at the shoulder on the non preferred side of the body. The projection of the ball machine was accurate within a circular area of $30 \mathrm{~cm}^{2}$. Participants caught either normal tennis balls or tennis balls specially made with an internal speaker emitting broadband sound ranging from $2500 \mathrm{hz}$ to $5000 \mathrm{hz}$ and the sound was randomised and pulsed at $75 \mathrm{db}$.

\section{Procedures}

Participants were split randomly into two experimental conditions. Group one was designated the control group $(n=11)$ non sound, group two was designated the experimental group $(n=9)$ broadband. Participants were instructed to catch the ball with their nonpreferred hand; this was the hand which they would not choose to write with. No other constraints were placed on the individuals.

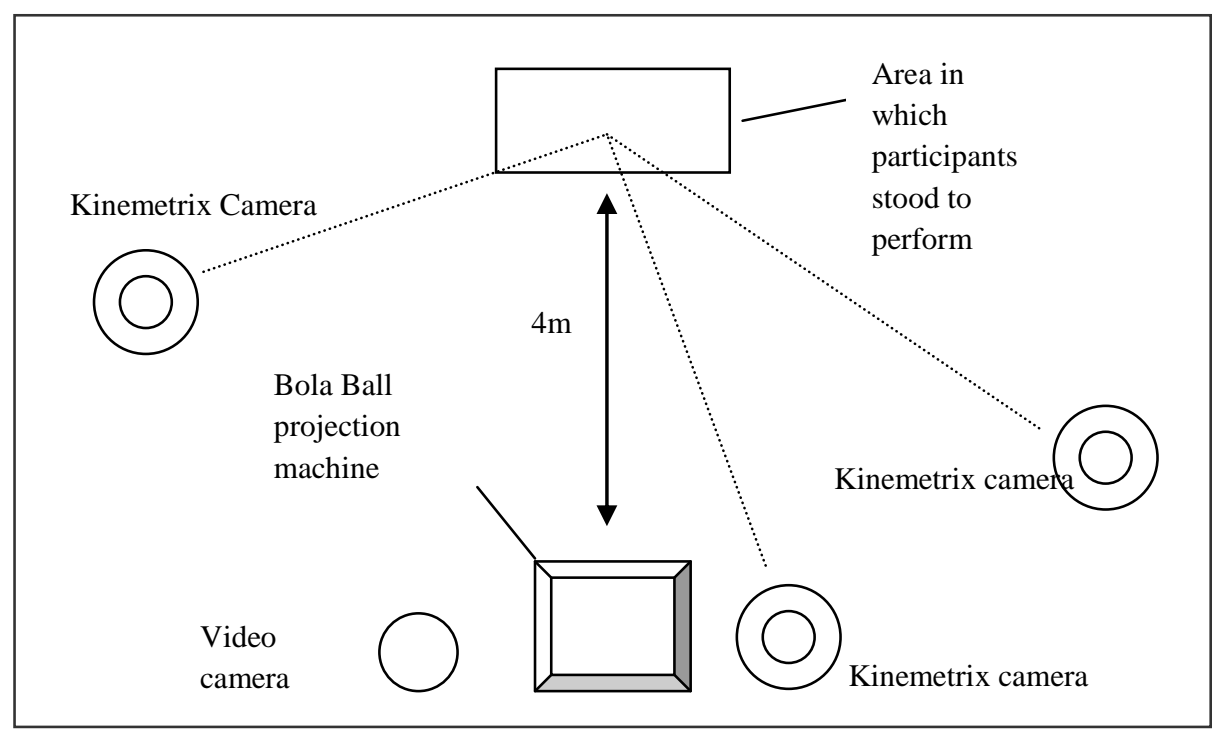

Figure 1 - Experimental set-up

Experimental data was collected at 3 blocks of trials. Experimental data was recorded both by the Kinemetrix system and a video camera throughout each trial block. After a familiarisation period of 2 catches, the prepractice trial block consisting of 10 balls, was recorded. A practice period of thirty balls either with a normal tennis ball (control group) or the broadband noise tennis ball (experimental group) followed, no kinematic data was recorded during this period. Immediately after the practice period, the post-practice trial block of 10 balls was recorded. The retention test, conducted a week after the initial trial consisted once again of a block trial of 10 balls and was recorded by the Kinemetrix system and a video camera. 
Analyses

\section{Video Analysis}

Video analysis of the 3 blocks of trials allowed qualitative analysis to be conducted on the number of balls caught by the participants. A catch was deemed successful based on a 0-5 point scale developed for use with young children and a two handed catch (Wickstrom,
1983, p163; see table1). As this experiment was conducted on adults performing a one handed catch, only a catch scoring 5 on the scale was deemed successful, meeting the criteria of: 'ball is contacted by the catching hands only and retained'. Analysis was completed by the author and an independent researcher. Interrater percent of exact agreements was $99 \%$ for this assessment tool, because of the high level of interrater agreement; the author's scores were used in all subsequent analysis.

Table 1- Description of catching behaviour in relation to points scoring scale of Wickstrom (1983, p193).

\begin{tabular}{|l|l|}
\hline $\begin{array}{l}\text { Points } \\
\text { Scored }\end{array}$ & Description of catching behavior \\
\hline 5 & Clean catch: ball is contacted by the catching hands only and retained \\
\hline 4 & Assisted catch: ball is juggled initially by the catching hands and then retained \\
\hline 3 & Hand contact: initial contact with the hand but the ball is subsequently dropped \\
\hline 2 & Upper body contact only and the ball is dropped \\
\hline 1 & Lower body contact only and the ball is dropped \\
\hline 0 & No ball contact with a part of the body \\
\hline
\end{tabular}

The number of successful catches, obtained from the qualitative assessment, was then quantitatively analysed. A $2 * 3$ (Group*Trial Period) mixed analyses of variance (ANOVA) with repeated measures was performed on the successful number of catches. The mean and standard deviation of the successful number of catches was also calculated. Eta Squared $\left(\eta^{2}\right)$

\section{Kinematic Analysis}

A 3D automatic kinematic analysis system was used to collect quantitative data of the nonpreferred arm of the participants. Infrared video technology automatically tracked and analysed the movement of reflective markers placed in the view of the cameras. After offline data screening, four subjects' data (3 from the control group and 1 from the sound ball group) were discounted owing to marker dropout. For remaining subjects, all data available was utilised in order to provide more accurate results where possible. The mean number of catching attempts per trial block from each individual which could be used was 6.6.

Peak velocities of the shoulder, elbow and wrist joints were measured along with the intralimb correlation between shoulder and elbow and calculations were conducted to assess the effect size of the results. A small effect size accounts for $1 \%$ to $3 \%(0.01-0.03)$ of the dependent variable associated with the independent variable, a medium effect accounts for 6-9\% (0.06-0.09) and a large effect explains $15 \%$ (0.15) or more (Cohen, 1977). The alpha level was set at .05 .

elbow and wrist. Correlations within limb velocity were proposed as a measure of intralimb coupling or the presence of coordinative structures. To assess the variability of the coordinative structure, the standard deviations of the correlations was calculated. Velocities of the limbs were correlated throughout the duration of the catch at each data point. The duration of the catch was calculated from the time at which acceleration began - when the wrist velocity increased for at least $4 \mathrm{~ms}$ - to the point at which, after a period of deceleration, a further acceleration period was noted. Using this measurement, the percentage time that the wrist spent in the acceleration phase of the catch was

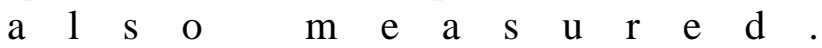

A 2*3 (Group*Trial Period) mixed analyses of variance (ANOVA) with repeated measures was 
performed on shoulder, elbow, and wrist peak velocity as well as on acceleration time and standard deviations of cross correlations. Prior to analyses of cross correlations, all correlation coefficients underwent a Fisher $z$ transformation. Eta Squared $\left(\eta^{2}\right)$ calculations were conducted to assess the effect size of the results. The affect sizes were assessed once again in accordance with Cohen (1977). The alpha level was set at .05 .

\section{Results}

\section{Video Data}

\section{Number of Successful Catches}

Figure 2 shows that the experimental group improved the mean number of successful catches across all three blocks of trials. The control group demonstrated an improvement between pre-practice and post-practice, but this improvement was not maintained into the retention trial. Statistical analysis indicated that there was a main affect of trial block for successful catches $(\mathrm{F}(1,16)=8.17, p \leq .05$, $\left.\eta^{2}=.14\right)$, and no main affect of group $(\mathrm{p}=.05)$. A significant interaction between group and block of trial was not found, although the result was approaching significance: $(F(1,16)=3.38$, $\left.\mathrm{p}=.055, \eta^{2}=.238\right)$.

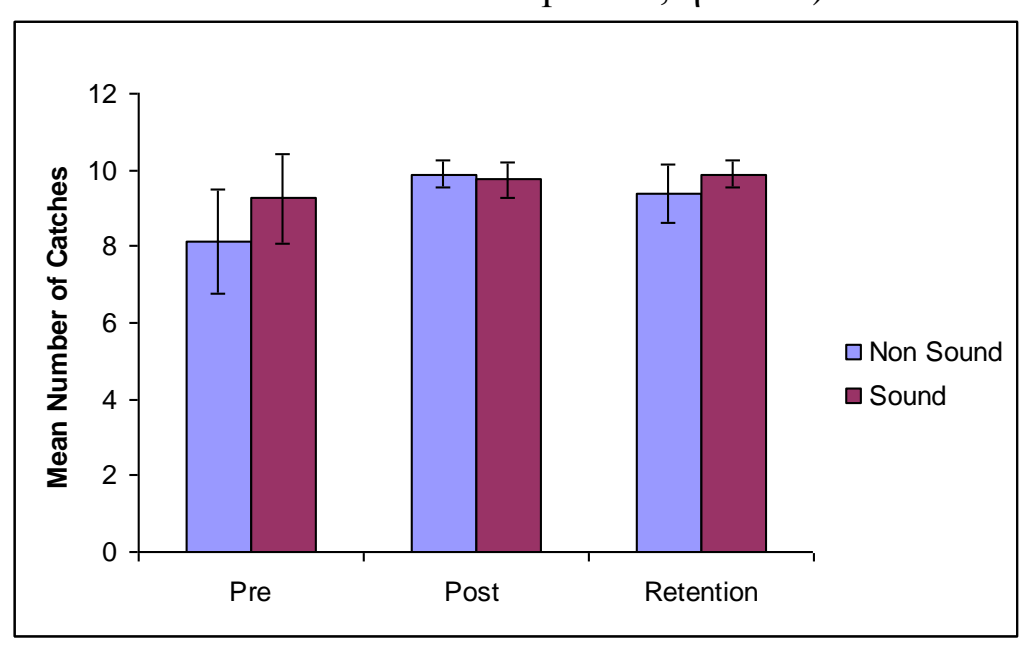

Figure 2 - Mean number of successful catches for each trial block for both the non-sound ball (control) and sound ball (experimental) groups.

\section{Kinematic Data}

\section{Peak Velocity}

The peak velocity of the shoulder, elbow and wrist of the control group showed an increase across each block of trial with a large increment seen in the wrist velocity in the retention test (see figure 3). This was not mirrored in the experimental group; the shoulder and elbow peak velocities showed an increase post-practice but subsequently decreased to a speed lower than recorded in the pre-practice block of trial (figure 4). Only the wrist velocity showed a consistent increase throughout all blocks of trials. Comparison of the experimental and control groups, shows that the shoulder velocities are similar in magnitude but the elbow and wrist peak velocities are higher in the experimental group, however, the difference in peak wrist velocity between the groups is not seen in the retention test. 


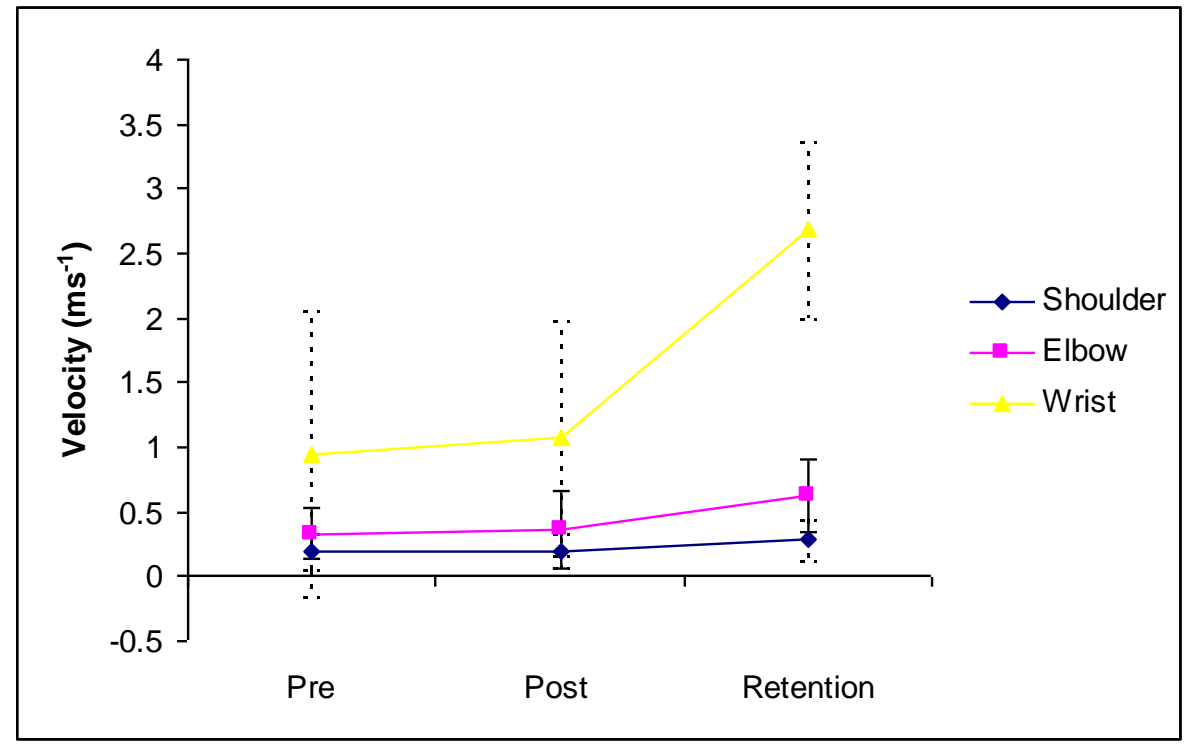

Figure 3 - Peak velocity of each joint across three blocks of trials in the control group (---error bars denotes the $S D$ of the wrist and shoulder velocity, - error bar denotes the $S D$ of the elbow velocity).

No main affect of trial was seen $(p \geq .05)$. Only and shoulder velocity (elbow, $F(1,16)=2.93$, the elbow peak velocity elucidated a main affect of group, $(F(1,16)=16.43, p \leq .001)$. A significant main affect of group is approached in the peak wrist velocity, $(F(1,16)=3.99$, $\left.\mathrm{p}=.066, \eta^{2}=.22\right)$ but not in peak shoulder velocity, $\left(F(1,16)=1.13, p \geq .05, \eta^{2}=.08\right)$. Statistical analysis showed a significant interaction approaching significance for elbow $\mathrm{p}=.07, \eta^{2}=.17$; and shoulder, $F(1,16)=2.66$, $\mathrm{p}=.087, \eta^{2}=.16$.) and a significant interaction between group and trial block of the wrist velocity $\left(F(1,16)=12.3, p \leq .05, \eta^{2}=.38\right)$. This is due to more than a doubling of the peak wrist velocity of the control group in the retention test (figure 3).

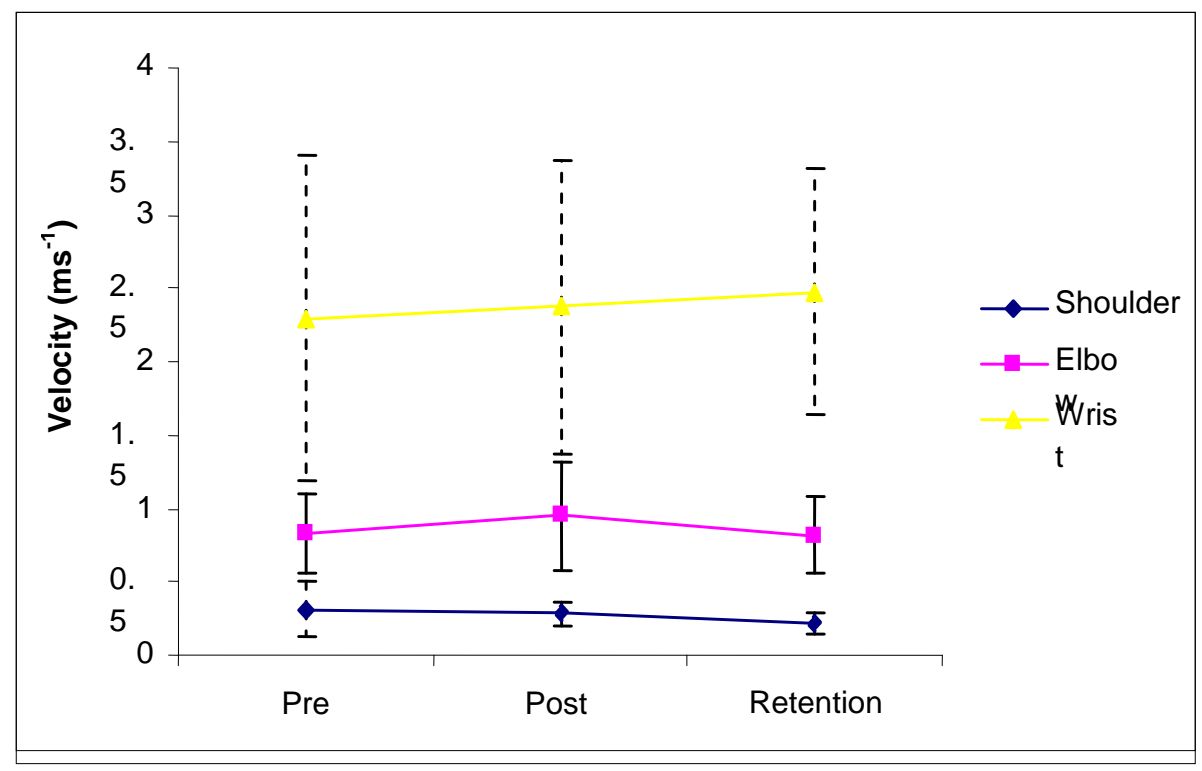

Figure 4 - Peak velocity of each joint across three blocks of trials of the experimental group (--error bars denotes the $S D$ of the wrist and shoulder velocity, - error bar denotes the $S D$ of the elbow velocity). 
The standard deviation of the wrist peak velocity is larger across all blocks of trials than in the elbow and shoulder joints (see figures $3 \&$ 4 ); this is an indication of a large variability of movement within each trial block. Both the experimental and control groups illustrate this trend.

\section{Wrist Acceleration Time}

Figure 5 shows that the experimental group (sound) increased the time spent accelerating between pre-practice and post-practice but this was markedly reduced in the retention test. Both the post-practice and retention test showed a very high standard deviation (see table 2). Conversely, the control group (non sound) substantially decreased the time the wrist spent in the acceleration phase of the catch between pre- and post-practice with this change being maintained into the retention test. A large standard deviation was seen in the post test but this value decreased in the retention test to a value similar to that of the pre-practice test (see table

2).

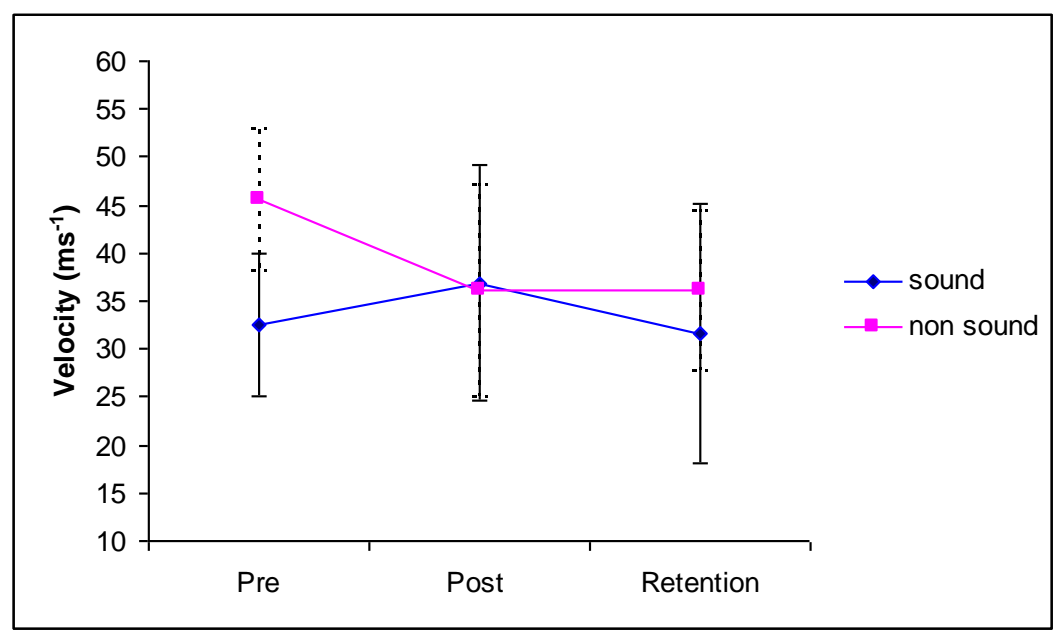

Figure 5 - Percentage of time the wrist spent in the acceleration phase of the catch (---- error bar denotes $S D$ of the non sound ball group, - error bar denotes $S D$ of soundball group).

Statistical analysis of the percentage wrist acceleration time indicated that there was no main affect of group or trial $(\mathrm{p}=.05)$ and a significant interaction between group and block of trial, $\left(F(1,16)=3.97, p \leq .05 ., \eta^{2}=.22\right)$. This significant interaction is due to a large decrease in the percentage wrist acceleration time seen in the control (non sound) group from pre- to post- practice trials, to a level equal to that of the experimental (sound) group. Furthermore, group comparisons of standard deviations of the mean acceleration time showed no main affect of group or trial and that a significant interaction between group and trial block was approached, $\left(F(1,16)=2.86, \mathrm{p}=0.75, \eta^{2}=.17\right)$.

Table 2 - Standard deviation for the percentage wrist acceleration time for sound (experimental) and non sound (control) groups.

\begin{tabular}{lll}
\hline \multicolumn{2}{l}{ Wrist Acceleration Time $(\%)$} & \\
\hline \multirow{3}{*}{ Pre } & Group & Std. Dev \\
& Sound & 7.43 \\
\multirow{2}{*}{ Post } & Non Sound & 6.86 \\
& Sound & 12.26 \\
Retention & Non Sound & 10.92 \\
\hline
\end{tabular}




\section{Mean Intralimb Correlation}

The experimental group (sound) showed a decrease in mean correlation across all blocks of trials in the shoulder-elbow correlation with the retention test mean correlation being almost half of the pre-practice value. Conversely, the elbow-wrist mean correlations of the experimental group showed little difference Table 3 - Standard deviation of the mean cross correlations. between pre- and post-practice and an increase in the retention test. Figure 6 clearly shows this and it appears that as the elbow and wrist form a more tightly coupled coordinative structure, the shoulder and elbow begin to move more independently of each other.

\section{Average Correlations}

\begin{tabular}{llll} 
& & Shoulder-Elbow & Elbow-Wrist \\
Pre & Group & Std. Dev & Std. Dev \\
\multirow{3}{*}{ Post } & Sound & 0.31 & 0.31 \\
\multirow{3}{*}{ Retention } & No Sound & 0.33 & 0.16 \\
& Sound & 0.35 & 0.59 \\
& No Sound & 0.35 & 0.42 \\
& Sound & 0.36 & 0.37 \\
\hline
\end{tabular}

The control group (non sound) demonstrated a coordinative structure. The mean elbow-wrist small increase in average correlation of both shoulder-elbow and elbow-wrist immediately after practice, which was not maintained into the retention test. In contrast to the experimental group, the correlations between joints of the control group mirrored each other (figure 6); this is an indication that the whole cross correlations of the control group were less than half the value of the experimental group. Standard deviations of the average correlations remained low for both groups across each trial with the greatest differences seen between the two groups in the elbow-wrist standard deviation (see table 3). arm was more tightly coupled to form a

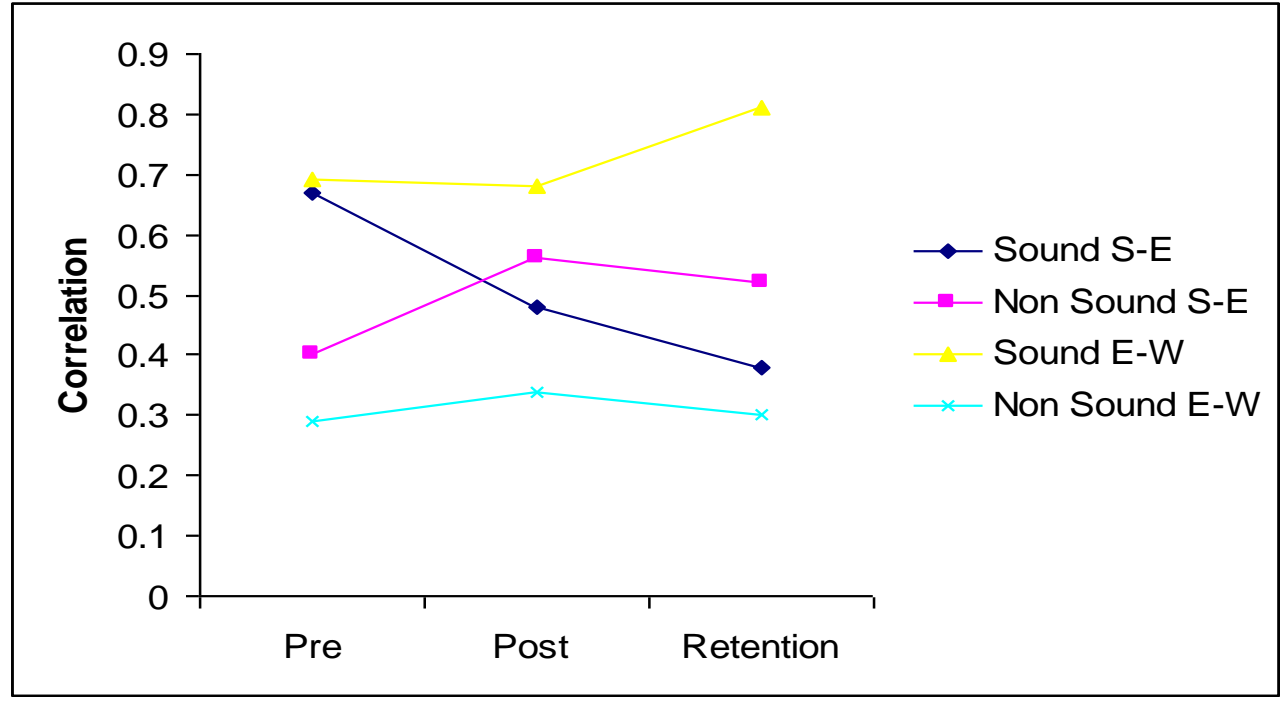

Figure 6 - Mean cross correlations between joints of both groups across three blocks of trials. (S-E $=$ shoulder and elbow, $\mathrm{E}-\mathrm{W}=$ elbow and wrist) 
Statistical analysis of this data indicated no main affect of group for the shoulder-elbow average correlations; $(F(1,16)=1.80, p \geq .05$, $\eta^{2}=.11$ ) and a main affect of group for the elbow-wrist mean correlations; $(F(1,16)=8.45$, $\left.p \leq .05, \eta^{2}=.38\right)$. This can be seen clearly in figure 7; across all three blocks of trials, the mean elbow-wrist correlation of the control group (non sound) is less than half than the elbow-wrist mean cross correlation of the experimental (sound) group. The large effect size reveals that the difference was due to the experimental conditions. No main affect of trial was seen $(p=.05)$. Despite the above observations, no significant interaction between group and trial block was seen, ( $F$ at least 2 , $\left.p \geq .05, \eta^{2}=.03-.11\right)$.

\section{Intralimb Correlation Standard Deviation}

The standard deviation of the intralimb correlation for each catching attempt of each participant, was used as a measure of variability of movement. The experimental group showed an almost doubling of the standard deviation of the shoulder-elbow correlations between preand post-practice trials, with the retention test value decreasing but not as low as the prepractice trial block (see figure 7). This is not seen in the elbow-wrist standard deviation correlation which remained similar between preand post-practice with a decrease in the retention test. The large increase in the standard deviation of the shoulder-elbow standard deviation correlation was not seen in the control group who conversely illustrated a decrease in the standard deviation pre- to post-practice trial block, however this change is not maintained; the retention test value is very similar to the prepractice value. Similarly to the mean cross correlations, the control group illustrated a mirroring of the standard deviation of the cross correlation (see figure 7).

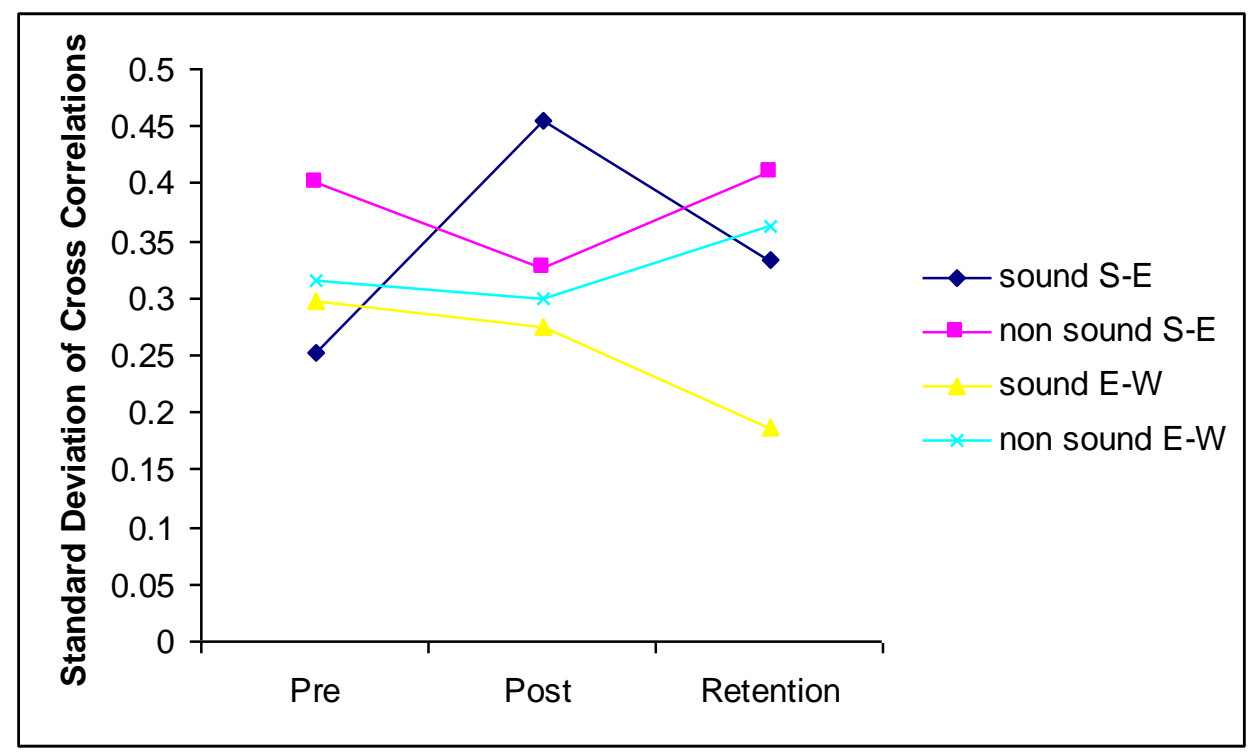

Figure 7 - Standard deviation of cross correlations between joints of both groups across three blocks of trials. (S-E = shoulder and elbow, E-W= elbow and wrist)

Statistical analyses indicated that there were no main affect of group or block of trial for either shoulder-elbow or elbow-wrist correlation standard deviations, $(F(1,16)$ at least 1.88 , $\left.p \geq .05, \eta^{2}=.03-.12\right)$. Within the shoulder-elbow correlation standard deviation, a significant interaction between group and trial block was seen, $\left(F(1,16)=3.67, p \leq .05, \eta^{2}=.21\right)$; this was not the case in the elbow-wrist correlation
$\left(F(1,16)=1.28, p \geq .05, \eta^{2}=.08\right)$. The interaction between the shoulder-elbow correlation standard deviation can be seen in figure 7 . The correlation standard deviation of the experimental group illustrates a large increase between pre- and post- practice trials, whereas the correlation standard deviation of the control group illustrates a large decrease between the same two trials. Furthermore, this opposing 
pattern is also seen between the post-practice and retention test, the experimental group decreasing and the control group increasing the shoulder-elbow cross correlation standard deviation.

\section{Discussion}

This study examined the intralimb correlation and joint coupling of expert catchers in a onehanded catching task. It is acknowledged by the authors that the size of the sample is relatively small and also that owing to the nature of the task a ceiling effect was evident. Catching a ball with the non-preferred hand is a simple task for adults with advanced movement control and experience. No participant screening was undertaken prior to experimentation. The success of the participants in catching the balls in the pre-practice test illustrates the level of their catching expertise (see figure 2). According to Davids, Bennett, Kingsbury, Jolly \& Brain (2000), all participants could be considered 'good' catchers, fulfilling the criteria of catching $70 \%$ of balls. This would mean that it is unlikely that such a short practice period 30 balls - would produce significant differences in the post-practice and retention test. If this experiment was conducted on younger children who were not so familiar with a one-handed catch results may yield more conclusive findings.

\section{Video Data}

The first observation arising from the results of this experiment is that the experimental group with augmented perceptual information, that is broadband noise, improved their catching performance across all blocks of trials (see figure 2). The control group however illustrate an improvement in catching performance between pre- and post-practice blocks of trials but this improvement is not maintained into the retention test conducted a week after the practice period. It can be proposed that this improvement is due to the enhancement of the perception-action process due to a more intense stimulation of the superior colliculus.

\section{Kinematic Data}

\section{Peak Velocity}

The experimental group illustrate more clearly that the movement of the catch is carried out at the hand; the shoulder and elbow peak velocities decrease in the retention test to a level lower than the pre-practice trial but the wrist peak velocity increases across all three trials (see figure 4). The control group, who practiced without additional environmental stimuli, illustrated a large increase in peak wrist velocity in the retention test suggesting that it is only in the final block of trials that the movement of the catch is predominantly carried out at the hand (see figure 3). Individual qualitative analysis of the control group would be of benefit here to analyse if a different catching behaviour was seen in the retention test.

The large standard deviation of the wrist peak velocities of both the experimental and control groups in comparison to that of the elbow and shoulder is an implication of variability of movement within trial (see figures $3 \& 4$ ). If subjects 'play' with the orientation of the hand, this would result in a larger standard deviation. This suggests that the gross positioning of the arm is more consistent than the grasp and grip stage of the catch.

\section{Wrist Acceleration Time}

It has been found that the effects of increasing spatial precision in a reaching and grasping task leads to a greater proportion of the movement time being spent in the deceleration phase (Roy, Rohr \& Weir, 2004). Catching a fast ball can be viewed similarly to a reaching and grasping task as they are both interceptive tasks requiring a great deal of spatial precision particularly in the grasp phase. If this is the case, then novices and unskilled catchers would require a greater time in the deceleration phase of the catch than experts. The results clearly illustrate that wrists of the experimental group across all three blocks of trials spend more time accelerating whereas conversely the control group decreased time accelerating, hence prolonging the length of the deceleration phase (see figure 5). This can be explained by the addition of auditory information available to the experimental group 
throughout the practice period. This may have enhanced their perceptual-action coupling resulting in an impression that the task goal presented to them was easier hence affording them to spend less time decelerating prior to initiating the catch. If broadband noise allows participants to localise sound in space (Knudsen et al, 1987) it would provide more information for action and therefore it would be easier for the experimental group to achieve the fine orientation demands of the grasp stage of the catch (Alderson et al, 1974) thus requiring less time in the deceleration phase. This could possibly lead to a shortened movement time.

\section{Intralimb Correlations}

The control group increased the average correlation of both the shoulder-elbow and elbow-wrist between pre- and post-practice trials (see figure 6); this is an indication of the formation of coordinative structures which is suggestive of Bernstein's first stage of the control process. This along with the evidence that the peak velocity of the wrist increases far more than the shoulder or the elbow in the retention test (see figure 3), allows the conclusion to be drawn that after practice the movement of the catch is predominantly carried out at the hand, there was little need to move the whole arm to successful carry out the task goal. Indicating that with practice the hand is pre positioned for the duration of the catch.

A large standard deviation of the intra-limb correlation would indicate flexibility of movement. The experimental group showed an increase in the standard deviation of the intralimb correlations across trials illustrating a large variation in movement (see figure 7). An explanation for this has been proposed by Fischman, Moore \& Steele (1992) who suggest that as children get older and become more skilful, they are able to 'play' with the way they respond when a ball is tossed towards them. When the task is relatively simple, participants have more freedom to experiment with their responses to achieve a task goal. As this task was simple for the participants, manipulation and experimentation of their movements would have made the task more interesting. This finding along with the discovery that there was a large standard deviation of the wrist peak velocities of both the experimental and control groups in comparison to that of the elbow and shoulder, as previously discussed, are implications of variability of movement. Furthermore, no constraints were placed upon the participants prior to testing, the instruction they were given was simply to try to catch the ball with their non-dominant hand; this seemingly resulted in great variation in movement.

\section{General Discussion and Conclusion}

As this study investigated the affect of broadband noise upon catching behaviour, it is pertinent to analyse the amount of time that the additional information was available for processing. If the ball machine had been placed at a greater distance from the subject, there would have been more time for sensory processing and enhancement of the perception action coupling which may have resulted in greater differences between the control and experimental groups. In previous studies, distances of $5.2 \mathrm{~m}, 7 \mathrm{~m}, 8 \mathrm{~m}$ and $13 \mathrm{~m}$ have been used (Bennett, Davids \& Woodcock, 1999; Tayler \& Davids, 1997; Smyth \& Marriott, 1982; Lefebvre \& Reid, 1998). There is evidence that a common optimal movement pattern does not exist, particularly in highly skilled performers (Button, 2002). Further qualitative assessment of catching behaviour would allow individual profiling of the coordination patterns assembled by each participant and additional group differences may be observed.

There is large scope for future research into this area. An assessment of whether or not it is broadband noise which causes the differences between groups or if presenting an experimental group with noise of a single frequency would have the same affect. If the experiment was conducted on unskilled catchers with immature movement experience, there is a possibility that greater affects may be discovered.

The findings of this experiment suggest that the effect of broadband noise presented temporally 
and spatially coincident result in a greater affordance for action. This results in less time required in the deceleration phase of the catch and a lesser need for the formation of coordinative structures as the participants were able to 'play' with their movement patterns in order to achieve the task goal. The affects of catching the broadband noise ball on coordination and control were maintained into the retention test but this was not found in the control group, suggesting that skilled participants can make use of different types of information to support catching behaviour and can flexibly re-assembly the perception-action coupling in order to achieve the task goal.

\section{References}

Alderson, G.J.K., Sully, D.J. \& Sully H.J. (1974). An operational analysis of a onehanded catching task using high speed photography. Journal of Motor Behavior, 6, 217-226.

Bennett, S., Davids, K. \& Woodcock, J. (1999). Structural organization of practice: effects of practicing under different informational constraints on the acquisition of one-handed catching skill. Journal of Motor Behavior, 31, 3-9.

Bernstein, N.A. (1967). The Coordination and Regulation of Movements. Oxford: Pergamon Press.

Button , C. (2002). Auditory information and the co-ordination of one-handed catching. In Davids, K., Savelsberg, G., Bennett, S.J. \& Van der Kamp, J. (Eds) Interceptive Actions in Sport: Information and Movement. (pp 184194). London: Routledge.

Button, C., Davids, K., Bennett, S.J. \& Taylor, M.A. (2000). Mechanical perturbation of the wrist during one-handed catching. Acta Psychologica, 105, 9-30.

Carlile, S. \& King A.J. (1993). Auditory Neuroscience: from outer ear to virtual space, Current Biology, 3, 446-8.
Cohen, J. (1977). Statistical Power Analysis for the Behavioral Sciences. (Rev. ed.) New York: Academic Press.

Davids, K., Bennett, S., Court, M., Tayler, M.A. \& Button, C. (1997). The cognition dynamics interface. In Lidor, R. \& Bar-Eli, M. (eds) Innovations in Sport Psychology: Linking Theory and Practice. Netanya, Israel: ISSP.

Davids, K., Bennett, S., Kingsbury, D., Jolly, L. \& Brain T. (2000). Effects of postural constraints on children's catching behaviour. Research Quarterly for Exercise and Sport, 71, 69-73.

Fischman, M.G., Moore, J.B. \& Steele, K.H. (1992). Children's one-hand catching as a function of age, gender and ball location. Research Quarterly for Exercise and Sport, 63, 349-355.

Knudsen, E.I., du Lac, S \& Esterley, S.D. (1987). Computational maps in the brain, Annual Review of Neuroscience, 10, 41-65.

Lefebvre, C. \& Reid, G. (1998). Prediction in ball catching with and without a development coordination disorder. Adapted Physical Activity Quarterly, 15, 299-315.

Lewald, J., Ehrenstein, W.H. \& Guski, R. (2001). Spatio-temporal constraints for auditory-visual integration. Behavioral Brain Research, 121, 69-79.

Newell, K.M. (1991). Motor skill acquisition. Annual Review of Psychology, 42, 213-237.

Roy, E.A., Rohr, L.E. \& Weir, P.L. (2004). Effect of movement termination in single- and dual-phase pointing tasks. Motor Control, 8, 121-138.

Savelsbergh, G.J.P., Netelenbos, J.B., \& Whiting, H.T.A. (1991). Auditory perception and the control of spatially coordinated action in deaf and hearing children. Journal of Child Psychology and Psychiatry, 3, 489-500. 
Smyth, M.M. \& Marriott, A.M. (1982). Vision and proprioception in simple catching. Journal of Motor Behavior, 14, 143-152.

Stein, B.E. \& Meredith, M.A. (1993). The Merging of the Senses. Cambridge, MA: MIT Press.

Stein, B. E., Meredith, M. A., Huneycutt, W. S., Mcdade, L. Behavioral indices of multisensory integration: orientation of visual cues is affected by auditory stimuli. J. Cogn. Neurosci. 1989; 1: 12-24,

Tresilian, J.R. (1999). Analysis of recent empirical challenges to an account of interceptive timing. Perception and Psychophysics, 61, 515-528.

Turvey, M.T. (1977). Preliminaries to a theory of action with reference to vision. In Shaw, R. \& Bransford, J. (Eds) Perceiving, Acting and Knowing: Toward an Ecological Psychology, (pp 211-266). Hillsdale NJ: Erlbaum.

Utley A, Nasr M, Astill S (2010) The use of sound during exercise to assist development for children with and without movement difficulties. Disability and Rehabilitation. 32(18):1495-500.

Utley A,, Steenbergen B, Astill SL. (2007) Ball catching in children with developmental coordination disorder: control of degrees of freedom. Developmental Medicine and Child Neurology.; 49(1):34-8.

Utley, A and Astill S.L (2007) Developmental sequences of two-handed catching: How do children with and without developmental coordination disorder differ? Physiotherapy Theory and Practice, 23, 2 65-82

Vereijken, B., van Emmerick, R.E.A., Whiting, H.T.A. \& Newell, K.M. (1992). Free(z)ing degrees of freedom in skill acquisition. Journal of Motor Behavior, 24, 133-142.

Yi-Chuan Chen, Su-Ling Yeh. (2009) Catch the moment: multisensory enhancement of rapid visual events by sound. Experimental Brain Research 2009; 198:2-3,209-21 
Andrea Utley, M. Nasr, and A. Astill 